ARTICLE

DOI: $10.1038 / s 41467-018-04542-9$

\title{
Quantum limit transport and destruction of the Weyl nodes in TaAs
}

\author{
B.J. Ramshaw (1) 1,2, K.A. Modic ${ }^{3}$, Arkady Shekhter ${ }^{4}$, Yi Zhang ${ }^{1}$, Eun-Ah Kim (1) 1, Philip J.W. Moll (1) ${ }^{3}$, \\ Maja D. Bachmann ${ }^{3}$, M.K. Chan², J.B. Betts², F. Balakirev², A. Migliori², N.J. Ghimire ${ }^{5,2}$, E.D. Bauer (D) ${ }^{2}$, \\ F. Ronning ${ }^{2} \&$ R.D. McDonald (1) ${ }^{2}$
}

Weyl fermions are a recently discovered ingredient for correlated states of electronic matter. A key difficulty has been that real materials also contain non-Weyl quasiparticles, and disentangling the experimental signatures has proven challenging. Here we use magnetic fields up to $95 \mathrm{~T}$ to drive the Weyl semimetal TaAs far into its quantum limit, where only the purely chiral Oth Landau levels of the Weyl fermions are occupied. We find the electrical resistivity to be nearly independent of magnetic field up to $50 \mathrm{~T}$ : unusual for conventional metals but consistent with the chiral anomaly for Weyl fermions. Above $50 \mathrm{~T}$ we observe a two-orderof-magnitude increase in resistivity, indicating that a gap opens in the chiral Landau levels. Above $80 \mathrm{~T}$ we observe strong ultrasonic attenuation below $2 \mathrm{~K}$, suggesting a mesoscopically textured state of matter. These results point the way to inducing new correlated states of matter in the quantum limit of Weyl semimetals.

\footnotetext{
${ }^{1}$ Laboratory of Atomic and Solid State Physics, Cornell University, Ithaca, NY 14853, USA. ${ }^{2}$ Los Alamos National Laboratory, Los Alamos, NM 87545, USA

${ }^{3}$ Max-Planck-Institute for Chemical Physics of Solids, Dresden 01187, Germany. ${ }^{4}$ National High Magnetic Field Laboratory, Tallahassee, FL 32310 , USA.

${ }^{5}$ Argonne National Laboratory, Argonne, IL 60439, USA. Correspondence and requests for materials should be addressed to

B.J.R. (email: bradramshaw@cornell.edu)
} 
T he principle of emergence is beautifully demonstrated by the complex states of matter that arise in metals due to interactions. Conventional metals can become superconductors ${ }^{1,2}$, spontaneously break spatial symmetries ${ }^{3}$, emerge with fractionalized charge ${ }^{4}$, or even become insulating ${ }^{5}$. The recent prediction ${ }^{6,7}$ and discovery ${ }^{8,9}$ of Weyl semimetals brings a different ingredient to the table-Weyl fermions-that differ from conventional electrons in two key ways. First, they obey a linear or "massless" energy-momentum relationship, analogous to electrons in graphene but in three dimensions. Second, because of strong spin-orbit coupling and inversion symmetry breaking, spin "up" and "down" states are replaced by chiral "left" and "right"-handed states (Fig. 1) ${ }^{10}$. Can interacting Weyl fermions form states of matter that are unique from those that arise in conventional metals? Predictions include chiral excitonic $^{11}$ and density wave states ${ }^{12}$, topologically non-trivial superconductors $^{13,14}$, and Tomonaga-Luttinger liquids ${ }^{15}$, but no interacting states have been confirmed in Weyl semimetals to date. Both Weyl fermions and trivial quasiparticles exist in real Weyl semimetals, making it difficult to tell which effects are due to Weyl fermions and which effects can be ascribed to conventional semimetal physics ${ }^{16}$.

In addition to the possibility of new correlated states, Weyl semimetals exhibit a number of unusual non-interacting properties due to the chiral anomaly. Chirality, or "handedness", is a classically conserved quantity-the flows of left and right-handed quasiparticles (chiral currents) should be separately conserved. The correct quantum mechanical description of nature does not obey this symmetry, allowing for classically forbidden phenomena such as the decay of a neutral pion into two photons ${ }^{17,18}$. In the case of Weyl fermions it has been shown that parallel electric and magnetic fields can unbalance the number of left and righthanded Weyl fermions ${ }^{10}$, leading to exotic transport ${ }^{10,19-21}$ and optical $^{22}$ properties. Although this effect was originally predicted for a Weyl semimetal far in the quantum limit $(\mathrm{QL})^{10}$, most experiments probing these effects have focused on relatively low magnetic fields ${ }^{9,23-25}$. Again, interpretation of these results is hampered by the fact that there are multiple Weyl fermions and non-chiral quasiparticles all present at the Fermi energy.

In this work, by using high magnetic fields we are able to gap out the non-chiral quasiparticles in TaAs and move the chemical potential to the fully chiral $n=0$ Landau levels (LLs) belonging to the Weyl nodes. This allows us to directly observe the chiral anomaly in the QL, measure the opening of a gap in the $n=0 \mathrm{LLs}$ once the inverse magnetic length becomes longer than the momentum-space separation of the Weyl nodes above $50 \mathrm{~T}$, and discover the onset of a new state above $80 \mathrm{~T}$.

\section{Results}

High field transport in TaAs. Figure $1 \mathrm{~b}$ shows the resistivity of the Weyl semimetal TaAs in magnetic fields up to $95 \mathrm{~T}$. Immediately striking is the two-order-of-magnitude increase in resistance that onsets at $50 \mathrm{~T}$ for the current and field configuration $\mathbf{J}\|\mathbf{B}\| \mathbf{c}$ (note the logarithmic scale). In addition to its strong magnetic field dependence, $\rho_{z z}$ is also strongly temperature dependent above $50 \mathrm{~T}$. This is in contrast with the behavior at intermediate fields (between 7.5 and $50 \mathrm{~T}$ ), where $\rho_{z z}$ decreases and then saturates, becoming roughly temperature and field independent. We identify the transport behavior between 7.5 and $50 \mathrm{~T}$ as characteristic of the chiral anomaly in the QL for Weyl fermions, and the behavior above $50 \mathrm{~T}$ as indicative of mixing between left and right handed Weyl fermions. There are two features in the data that we do not believe to be inherent to the bulk transport of TaAs: a superconducting transition at low field, originating in $\mathrm{a} \approx 20 \mathrm{~nm}$ tantalum-rich amorphous layer induced during sample preparation; ${ }^{26}$ and the rollover of $\rho_{z z}$ at high field, either due to the same amorphous layer or due to the intrinsic surface states of $\mathrm{TaAs}^{9,27,28}$. Neither effect impacts the conclusions of this paper (see Methods).

We measure both $\mathbf{J}\|\mathbf{B}\| \mathbf{c}-\rho_{z z}$-and $\mathbf{J} \perp(\mathbf{B} \| \mathbf{c})-\rho_{x x}$-to identify different regimes in the electronic structure of TaAs (Fig. 2). TaAs contains three distinct types of quasiparticles: two electron-like Weyl Fermi surfaces (denoted W1 and W2 $2^{9,23,25,27,29}$ ), and a hole-like Fermi surface with a trivial spectrum ${ }^{23,29,30}$. Figure $2 \mathrm{c}$ highlights a key difference in the Landau quantization between Weyl and trivial carriers: the energy of the $n=0$ LL of Weyl carriers is field-independent and disperses linearly in $k_{z}$ (momentum parallel to B), whereas the energy of the $n=0 \mathrm{LL}$ of trivial carriers is field-dependent and disperses quadratically in $k_{z}$ This leads to qualitatively different behavior for the two carrier types in the QL, when only the $n=0 \mathrm{LL}$ is occupied ${ }^{31}$. As the magnetic field is increased TaAs passes through four distinct LL regimes, described in detail in the caption to Fig. 2. With the magnetic field aligned along the $c$-axis both the W1 and W2 Weyl pockets enter the QL (the chemical potential enters the 0th $\mathrm{LL}$ ) below $8 \mathrm{~T}$, as observed in both our data and in previous studies of the Fermi surface of $\mathrm{TaAs}^{29}$. The hole pocket enters the quantum a

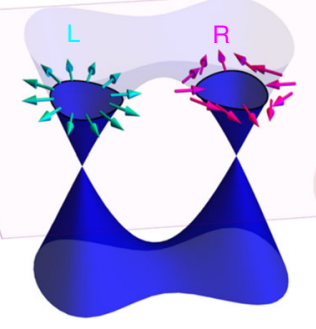

b

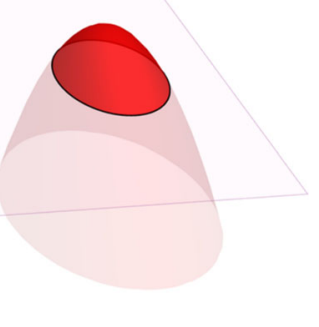

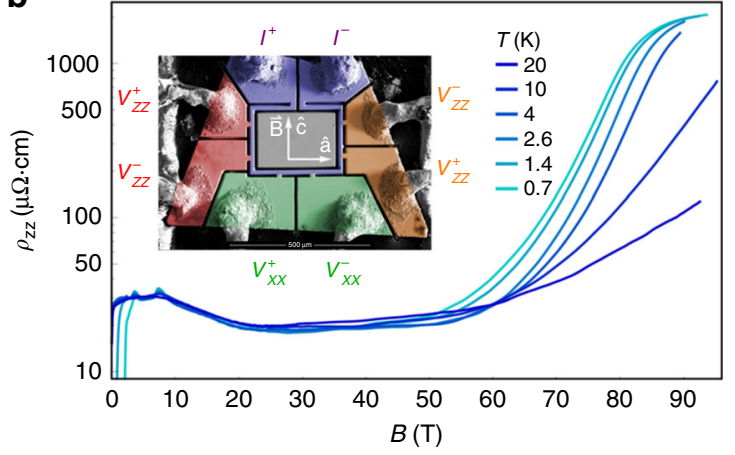

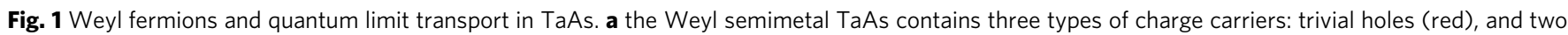
sets of electron-like Weyl fermions, designated W1 and W2, one of which is shown here in blue. The holes are of a single non-chiral carrier type, and contribute a non-Weyl background signature to experiments. The Weyl electrons are separated into distinct right and left-handed chiralities: arrows indicate the winding of the pseudospin around each Fermi surface. $\mathbf{b}$ Resistivity of TaAs for $\mathbf{J} \mid$ B $\|$ |c from 0.7 to $20 \mathrm{~K}$. Quantum oscillations from the Weyl pockets are visible up to $7.5 \mathrm{~T}$, followed by a decrease and then saturation of $\rho_{\mathrm{zz}}$ up to $50 \mathrm{~T}$. Above $50 \mathrm{~T}$ there is a two order-of-magnitude increase in $\rho_{z z}$ at low temperature, signifying the opening of a gap. The inset shows single-crystal TaAs microstructured using focused-ion-beam (FIB) lithography for both the $\rho_{z z}$ and $\rho_{x x}$ measurements 


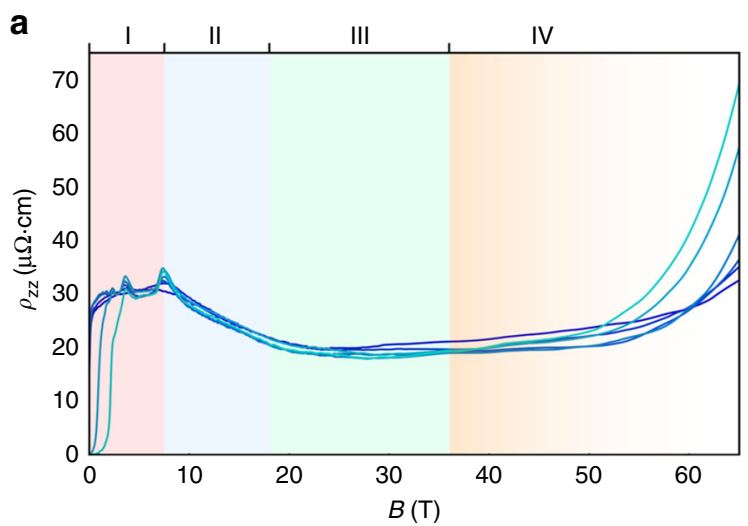

C
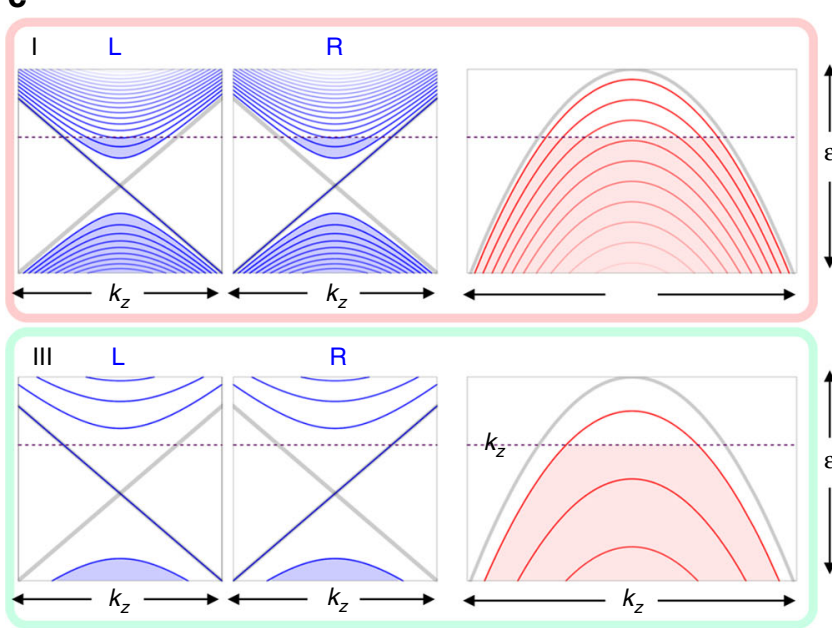

b

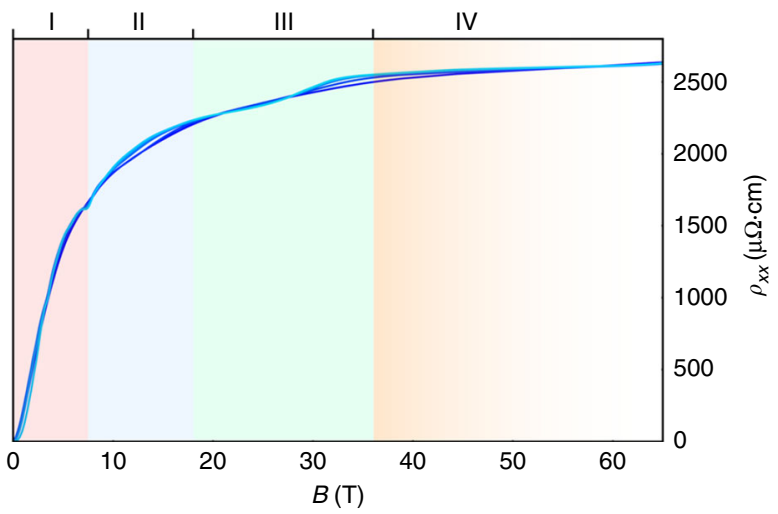

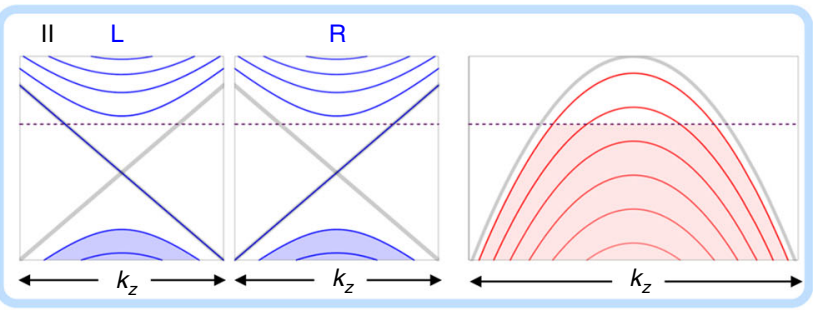

$\mathrm{R}$
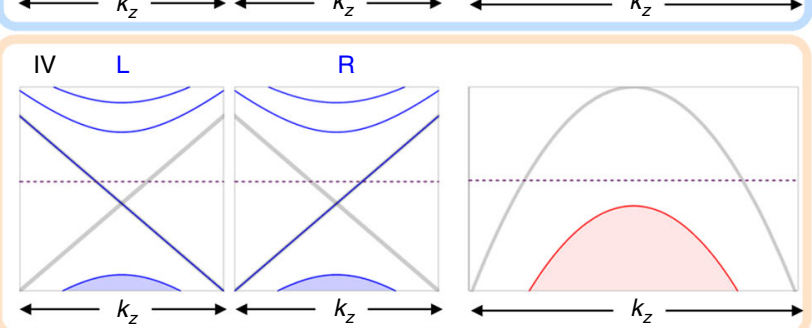

Fig. 2 The four distinct regimes of Landau level occupancy in TaAs up to $65 \mathrm{~T}$. TaAs hosts two pairs of Weyl fermi surfaces (W1 and W2) with similar quantum limits for this field orientation; one pair of Weyl nodes is shown here along with the trivial hole surface. Resistivity of TaAs at temperatures between $0.7 \mathrm{~K}$ and $20 \mathrm{~K}$, for $\mathbf{J}\|\mathbf{B}\| \mathbf{c}(\mathbf{a})$ and for $\mathbf{J} \perp(\mathbf{B} \| \mathbf{c})(\mathbf{b})$, from 0 to $65 \mathrm{~T}$. c , Schematic of the Landau level (LL) structure and occupancy for the parabolic hole pockets (red lines) and Weyl electron pockets (blue lines). Gray curves are the $B=0$ dispersions without LLs. Region $\mathbf{I}$ is from 0 to $7.5 \mathrm{~T}$, where both hole and electron pockets exhibit quantum oscillations. Between 7.5 and $18 \mathrm{~T}$ (region II) the electron pockets are in the $n=0 \mathrm{LL}$, whereas the hole pocket is still in the $n=1 \mathrm{LL}$. Between 18 and $36 \mathrm{~T}$ (region III) the hole pocket is also in the $n=0 \mathrm{LL}$. At 36 T the last oscillation, corresponding to emptying the $n=0$ $\mathrm{LL}$ of the hole pocket, can be seen in $\rho_{x x}$. Above $36 \mathrm{~T}$ (region IV) the only occupied states at the chemical potential are in the $n=0 \mathrm{LL}$ of the Weyl electron pockets, and the chemical potential shifts to maintain overall carrier number $\left(n_{\mathrm{e}}-n_{\mathrm{h}}\right)$. The faded region IV represents the mixing of the Weyl nodes above 50 T (Fig. 3)

limit at $18 \mathrm{~T}$ and is completely depopulated at $36 \mathrm{~T}$-also agreeing with previous studies ${ }^{29}$. The fact that the trivial hole carriers can be completely depopulated with field is a result of the field-independence of the $n=0 \mathrm{LL}$ of the Weyl pockets, where the chemical potential is pinned due to the small excess of electrons versus holes.

Chiral anomaly in the quantum limit. Above $36 \mathrm{~T}$ the chiral $n$ $=0$ LLs of the Weyl fermions can be studied without the complicating influence of trivial holes, and without the $n>0$ LLs for which chirality is not well-defined ${ }^{32}$. Each of the two 0th LLs of the Weyl fermions contains carriers propagating along a single direction-parallel to B for one chirality ("up movers"), and antiparallel for the other ("down movers"). Applying an electric field (driving current) parallel to $\mathrm{B}$ produces an imbalance in the number of up versus down movers, resulting in an imbalance of left and right-handed carriers-a chiral anomaly. This imbalance produces a chiral current, effectively increasing the conductivity as the electric and magnetic fields transfer charge from one Weyl node to the other ${ }^{10,19}$. The experimental signature of this phenomenon at low fields-negative magnetoresistance-has been controversial due to the presence of experimental artifacts ${ }^{32-34}$ and contributions from the trivial hole pocket. We find that using focused-ion-beam lithography (FIB) to reduce current-jetting can suppress or even eliminate the very low field negative magnetoresistance that has been reported for $\mathrm{TaAs}^{7,8}$ (see Methods), in line with an earlier study of current-jetting ${ }^{34}$. We do observe negative magnetoresistance from 7.5 to $25 \mathrm{~T}$ which may be related to the chiral anomaly, although not all samples show this to the same degree and this effect may depend sensitively on sample alignment (see Supplementary Methods). Our focus is not on the low-field regime but instead on the QL where the predicted behavior of the chiral anomaly is qualitatively distinct ${ }^{20}$.

In the QL, where transport along the $z$ direction is determined by the $n=0 \mathrm{LL}$ of the Weyl fermions alone, the conductivity has the form

$$
\sigma_{\mathrm{zz}}=N_{\mathrm{W}} \frac{e^{2} v_{\mathrm{F}}}{4 \pi \hbar l_{B}^{2}} \tau_{\text {inter }}(B),
$$

where $N_{\mathrm{w}}$ is the number of Weyl points in the Brillouin zone, $v_{\mathrm{F}}$ is the Fermi velocity, $l_{B}=\sqrt{(h / e B)}$ is the magnetic length, and $\tau_{\text {inter }}(B)$ is the field-dependent inter-nodal scattering time ${ }^{10,20}$. As 

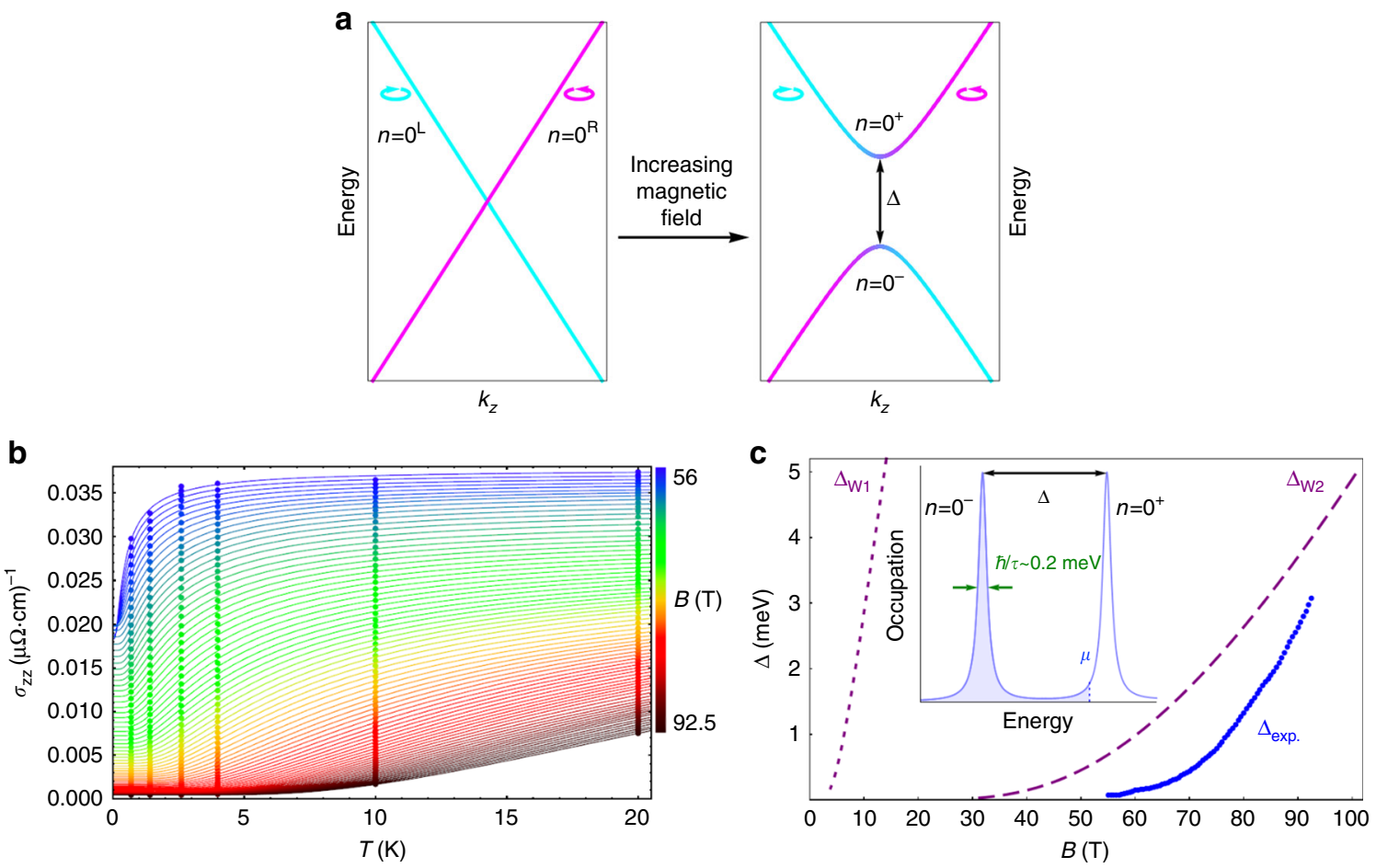

Fig. 3 Mixing of left and right-handed Landau levels in a magnetic field. The left and right-handed Weyl nodes are separated in the $k_{x}-k_{y}$ plane in TaAs (Fig. 1a) but are degenerate along $k_{\mathrm{z}}$. At low magnetic fields the left and right-handed $n=0$ LLs can be considered independent (a). As the magnetic field is increased, and the magnetic length approaches the inverse momentum-space separation of the nodes, the two LLs hybridize and a gap $\Delta$ opens that increases with field. We extract this gap by fitting the conductivity to $\sigma_{z z}=\sigma_{0}+\sigma_{1} e^{-\frac{\Delta}{k B T}}$ from 56 to $92.5 \mathrm{~T}$ (b, points are data and solid lines are fits at different values of the magnetic field), with $\Delta$ show in panel c. We find that the W1 nodes gap at low field due to their close momentum-space proximity $7,23,25,27$, whereas the much larger separation of the W2 nodes means that their gap opens at higher field $(\mathbf{b})$. The $\approx 0.5$ meV offset between our calculated and measured gaps may be due to the $\approx 0.2 \mathrm{meV}$ LL broading due to finite quasiparticle lifetime (inset)

shown below, the W1 Weyl nodes are most likely gapped in this field range (we use the notation of Arnold et al. ${ }^{29}$ for W1 and W2, opposite that of Lv et al. ${ }^{27}$ ). We therefore evaluate Equation 1 for the eight W2 Weyl nodes, using $v_{\mathrm{F}}$ determined by the quantum oscillations (see Methods). At $27 \mathrm{~T}$, where $\sigma_{\mathrm{zz}}$ is maximum, we calculate $\tau_{\text {inter }}=6 \mathrm{ps}$. This is $\sim 10$ times longer than the intranodal scattering time that we calculate from the Dingle factor of the quantum oscillations (see Methods), confirming that the chiral 0th LLs carry current with a reduced scattering rate in the QL due to their physical separation and distinct chiralities.

As the magnetic field is increased $\mathrm{TaAs}$ shows relatively constant resistivity in the QL before $50 \mathrm{~T}$ (Fig. 1b), requiring that the scattering rate $1 / \tau$ increases roughly in proportion to $B$ to cancel the factor of $l_{B}^{2}$ in Equation 1 . Indeed, $1 / \tau \propto B$, and the resulting field-independent conductivity, was predicted for the QL of Weyl semimetals assuming short-range impurity scattering, suggesting that this is the dominant scattering mechanism between the Weyl nodes in $\mathrm{TaAs}^{20}$. As pointed out by Spivak and Andreev ${ }^{20}$, short-range impurity scattering in the quantum limit of conventional semimetals leads to longitudinal magnetoresistance that increases strongly with magnetic field ${ }^{35,36}$. Thus our observation of relatively constant $\rho_{z z}$ up to seven times the QL is a unique property of Weyl fermions.

Gap formation in the chiral $\boldsymbol{n}=\mathbf{0}$ Landau levels. Having established the QL transport properties of TaAs at intermediate fields we turn to higher fields where $\rho_{z z}$ increases rapidly above $50 \mathrm{~T}$ (Fig. 1b). While the 0th LLs of the Weyl nodes should naively remain metallic in the QL, the two-order-of-magnitude increase in $\rho_{z z}$ suggests the formation of a gap. Weyl nodes are topologically stable to small perturbations ${ }^{16}$, but this can be overcome by strong magnetic fields which mix the left and righthanded $n=0$ LLs (Fig. 3a). If the chemical potential falls within the gap then thermal activation is required to promote charge carriers to the empty LL, resulting in a strong temperature dependence for $\rho_{z z}$. As pointed out previously ${ }^{37-39}$ a gap in the $0^{\text {th }}$ LLs should be observable when the inverse magnetic length $1 / l_{B}=1 / \sqrt{(\hbar / e B)}$ - which controls the momentum-space extent of $0^{\text {th }}$ LL wavefunctions-becomes large compared to the momentum-space separation of the Weyl nodes. This mechanism implies that the left and right handed chiral quasiparticles lose their distinction when their wavefunctions overlap, allowing for increased scattering between them and the formation of a gap. This is the same mechanism proposed for the observed change in sign of the Hall coefficient in $\mathrm{TaP}^{39}$. The significant difference between TaAs and $\mathrm{TaP}$ is that one pair of Weyl nodes in $\mathrm{TaP}$ remains ungapped up to the highest measured fields, whereas all nodes are eventually gapped in TaAs. The high sensitivity of $\sigma_{\mathrm{zz}}$ to the opening of the gap in TaAs allows us to produce a highly accurate measure of Weyl node separation in the bulk material.

To quantify this picture we extract the experimental gap by fitting the temperature-dependent conductivity at different values of the magnetic field where the gap opens above $56 \mathrm{~T}$ (Fig. 3b). The data are well-described by the model $\sigma_{z z}=\sigma_{0}+\sigma_{1} e^{-\frac{\Delta}{k_{\mathrm{B}} T}}$, where $\sigma_{0}$ is a field-dependent background, $\sigma_{1}$ is the conductivity from the $0^{\text {th }}$ LLs, and $\Delta$ is the gap (plotted as $\Delta_{\text {exp }}$ in Fig. $3 c$ ). This model assumes that the gap dominates the field-dependence of the transport and we keep the scattering rate fixed as function of field for simplicity. To relate this gap to the electronic structure of the Weyl nodes we developed a complete tight binding model of 
a

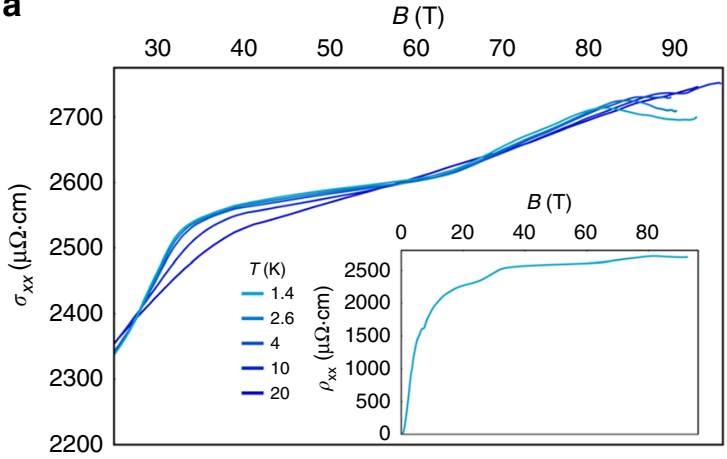

b
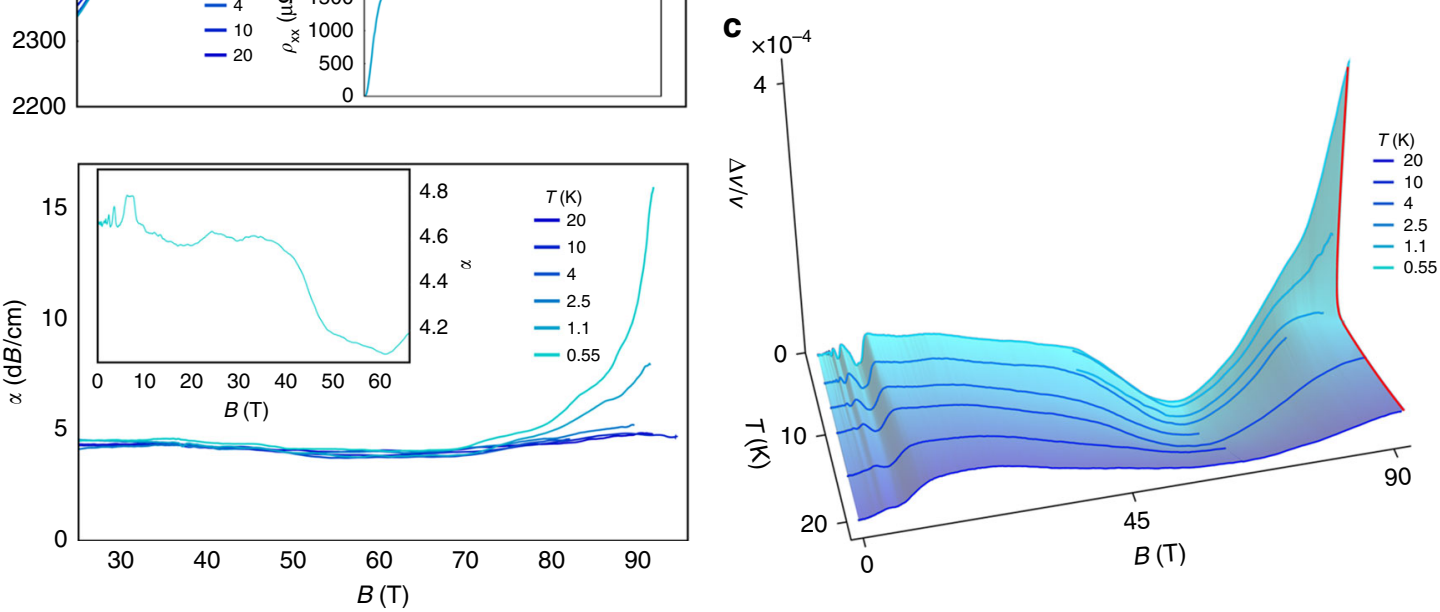

Fig. 4 Phase transition in the quantum limit of TaAs. a Resistivity for $\mathbf{J} \perp(\mathbf{B} \| \mathbf{c})$, showing a kink near $80 \mathrm{~T}$ at low temperature. $\mathbf{b}$ The ultrasonic attenuation at $315 \mathrm{MHz}$ for $\mathbf{k}\|\mathbf{B}\| \mathbf{c}$, where $\mathrm{k}$ is the propagation wavevector of the longitudinal (compressional) sound, and with data from 0 to $65 \mathrm{~T}$ shown in the inset. $\mathbf{c}$ Change in the speed of sound for the same configuration as the attenuation shown in (b). Data from two different experiments-one from 0 to $65 \mathrm{~T}$ and one from 45 to $95 \mathrm{~T}$-are combined in this plot. Above $2.5 \mathrm{~K}$ the sound velocity flattens out above $80 \mathrm{~T}$ and the attenuation is only weakly field dependent. Below $2.5 \mathrm{~K}$, however, both the sound velocity and the ultrasonic attenuation increase rapidly with field. The red line is an interpolation of the data, highlighting the abruptness of the transition as a function of temperature

TaAs in a magnetic field, including all 24 Weyl nodes and Zeeman coupling (see Methods for a full description of the tight binding model and LL calculations). Fixing $v_{F}$ to the experimentally determined value, we find that $\delta k=0.15 \pi / a$ gives the correct slope of $\Delta$ versus $B$ at high field. The slope of $\Delta$ versus $B$ is a strong function of $\delta k$, and therefore provides a bulk-sensitive measurement of the Weyl node separation. Our extracted value of $\delta k$ also fits the range of W2 node separation estimated by photoemission measurements (between $0.12 \pi / a$ and $0.15 \pi / a)^{9,27}$. The W1 nodes are estimated to be separated by at most $\delta k=$ $0.04 \pi / a$, and are therefore gaped at much lower field (Fig. 3c).

It should be noted that perfect electron-hole compensation is required for the chemical potential to lie in the gap at high fields: excess of electrons (holes) will pin the chemical potential in the $n$ $=0^{-}\left(n=0^{+}\right)$LL to maintain the net carrier concentration (Fig. 3a). Hall effect measurements indicate that compensation in TaAs may not be perfect, with an estimated $2 \times 10^{17} \mathrm{~cm}^{-3}$ to $5 \times$ $10^{18} \mathrm{~cm}^{-3}$ excess electrons ${ }^{28,29}$ that naively should remain metallic at high fields. In reality once the magnetic length is short enough that the left and right nodes overlap scattering will increase greatly and eventually localize any residual carriers. The estimated excess electron density in TaAs is $\approx 10^{12}$ carriers per $\mathrm{cm}^{2}$-within the range of a metal-insulator transition ${ }^{40,41}$. This may explain why we observe the gap in $\rho_{z z}$ at slightly higher fields than both our model and the model of Chan:2017 predicts. Note that we do not observe a similar increase in $\rho_{x x}$ above $50 \mathrm{~T}$ : the in-plane conductivity has an $80 \mathrm{meV}(1000 \mathrm{~K})$ cyclotron gap at $50 \mathrm{~T}$, and in-plane transport in this regime is dominated by impurity assisted hopping between adjacent $n=0$ LLs in real space $^{36}$. Thus $\rho_{x x}$ should be weakly temperature dependent for $\mathbf{B} \| \mathbf{c}$, consistent with what we observe experimentally.
Phase transition in the quantum limit. At $80 \mathrm{~T}$, well into the gapped state, we observe a small decrease in $\rho_{x x}$ at the lowest temperatures (Fig. 4a)-possibly indicative of a phase transition. To investigate this possibility further we measured the sound velocity $\left(v_{z z}\right)$ of TaAs using pulse echo ultrasound up to $95 \mathrm{~T}$, providing a thermodynamic probe at the highest fields. Below 2.5 $\mathrm{K}$ and above $80 \mathrm{~T}$ we observe an increase in sound velocity accompanied by a strong increase in ultrasonic attenuation (Fig. $4 \mathrm{~b}, \mathrm{c}$ ). The onset of these ultrasonic features coincides with the sharp decrease in $\rho_{x x}$, and we take all three phenomena as evidence for a field-induced phase transition. Our numerical solutions of the tight binding model predict no further transitions as a function of magnetic field once the gap opens along $k_{z}$, suggesting that this transition at $80 \mathrm{~T}$ is driven by interactions.

There is precedent for an interaction-driven phase transition in the quantum limit of a semimetal. Graphite, whose quantum limit is $75 \mathrm{~T}$, undergoes a rich series of transitions above $30 \mathrm{~T}$. These transitions are generally ascribed to excitonic or Peirels-type density-wave formation along the magnetic field direction ${ }^{42-44}$. This mechanism seems unlikely to occur in TaAs given that a gap has already opened along $k_{z}$ at $50 \mathrm{~T}$. In the limit where the ultrasonic wavelength is much longer than the quasiparticle mean free path, attenuation is proportional to conductivity ${ }^{45}$. The fact that we observe attenuation to increase while $\sigma_{z z}$ decreases suggests that something other than conventional electronic attenuation is occurring above $80 \mathrm{~T}$ in TaAs. The enhanced attenuation could come from the interaction between ultrasound and order-parameter fluctuations, as it does in graphite where it peaks near each field-induced phase transition ${ }^{46}$. The unbounded growth in attenuation we observe suggests that either we have not 
yet reached the attenuation peak, or that a different attenuation mechanism is at work.

To speculate on the microscopic origin of the high-field phase it is helpful to consider which degree of freedom remains after the gap opens along $k_{z}$. The in-plane cyclotron orbits of the quasiparticles shrink in size with increasing magnetic field, resulting in a large real-space degeneracy of the $n=0$ LLs (or equivalently momentum-space overlap of the quasiparticle wavefunctions). This increases the Coulomb repulsion between quasiparticles as $E_{\mathrm{C}} \propto e^{2} / l_{B}$, ultimately leading to Wigner crystallization. Before this transition occurs, however, there are intermediate "mixed" or "microemulsion" phases with spatially phase-separated regions of Fermi liquid and bubbles of crystallized electrons ${ }^{47}$. Disorder pins these structures in real-space, resulting in a mesoscopically inhomogeneous system. This texture, with a length-scale comparable to the ultrasonic wavelength, is known to scatter ultrasound. Clearly this phase needs to be explored further before it can be identified: the frequency dependence of the attenuation would yield the length scale of the phase separation ${ }^{48}$ or the frequency-dependence of the order parameter fluctuations; ${ }^{49}$ the collective sliding motion of crystallized electrons can be revealed by nonlinear currentvoltage measurements ${ }^{50}$.

\section{Discussion}

The ideal Weyl semimetal contains only one pair of Weyl nodes, with large momentum space separation to make them robust against perturbations. Reality is more interesting: the W2 Weyl nodes in TaAs have a large separation compared to other materials $^{7}$, but there are 12 pairs of nodes in total plus the trivial hole pockets. Using magnetic fields to drive Weyl semimetals into their QL provides a clear path forward for studying "pure" Weyl physics, taking advantage of the fact that the $0^{\text {th }}$ LLs are fieldindependent. This greatly broadens the number of potential systems where Weyl fermions can be accessed: as long as the magnetic field is strong enough the chemical potential will always move to the chiral $0^{\text {th }}$ Weyl LLs in the quantum limit, even if the zero-field Fermi surface encompasses both nodes. Given that magnetic fields also increase the Coulomb interaction between quasiparticles (which can be particularly weak in semimetals due to the high Fermi velocities), the QL is also a promising regime for finding new states of matter formed from Weyl fermions.

\section{Methods}

Sample preparation. Negative magnetoresistance has been reported for a large number of candidate Dirac and Weyl semimetals ${ }^{16}$. The observation of decreasing resistance with increasing magnetic field for $\mathbf{J}|| \mathbf{B}$ has been taken as evidence of the chiral anomaly. Some of these measurements have been disputed because of extraneous effects that can also lead to an apparent negative magnetoresistance. In particular for TaAs, the highly anisotropic nature of the magnetoresistance means that current injected into the sample through point-like contacts does not necessarily move uniformly through the sample. This effect has been known for quite some time (see page 43 of Pippard ${ }^{33}$ ), and recent numerical simulations explored this in the context of Weyl semimetals by Reis ${ }^{34}$. The result is that the potential drop across a pair of voltage contacts may not reflect the total current injected into the sample, depending on where the contacts are placed. This can be overcome by avoiding point-like contacts and by preparing samples with high aspect ratios with current injection taking place far from the voltage measurement. Even a standard precaution such as contacting the entire ends of a sample to inject current homogeneously does not necessarily produce consistent results since microscopic contact to the sample can still be point like or at the very least inhomogeneous.

To illustrate this point for TaAs we prepared the sample shown in Supplementary Fig. 1. The current path is highlighted in purple; the "good" voltage contacts in red; the "bad" voltage contacts in green. The corresponding resistances, or rather the voltage drop across the contacts divided by the nominal current, are shown in the two right hand panels for $\mathbf{B}\|\mathbf{J}\| \mathbf{c}$ : the top panel corresponds to the green-shaded contacts offset from the current path; the bottom panel corresponds to the red-shaded contacts that are closer to the current path. It is immediately clear that a sizable "negative magnetoresistance" is visible for the green contacts at all temperatures, similar in character to that reported as evidence for a chiral anomaly in TaAs by Zhang et al. ${ }^{30}$ and Huang et al. ${ }^{23}$. In our exaggerated geometry the voltage drops to near-zero at high fields: clearly an unphysical result. The apparent increase in this effect at higher temperatures is due to the increase in resistance and decrease in resistive anisotropy at higher temperatures: it takes more magnetic field to isolate the current from the green voltage contacts at higher temperatures because the resistive anisotropy is lower. The red contacts, on the other hand, show positive magnetoresistance that saturates at $\sim 1 \mathrm{~T}$-this is expected as $\omega_{c} \tau$ reaches 1 near this field value. We used an even more restricted current path geometry for the data presented in the main text, and a second sample with similar current path characteristics shows similar resistivity in Supplementary Fig. 3.

$\rho_{z z}$ shown in Fig. 1 of the main text show signs of saturation at the highest resistance values. This may be due to a parallel conduction channel on the surface of the sample. There are two possible sources of this conduction channel: the surface states which are known to be present on this material and which have been observed via ARPES; ${ }^{9}$ and/or an amorphous arsenic-depleted layer induced by the $\mathrm{FIB}^{26}$.

As can be clearly seen in the inset of Fig. 1 in the main text, the FIB prepared samples undergo a superconducting transition at low temperatures and low magnetic field. This is also a surface effect: it was recently shown that after FIB microstructuring the related material $\mathrm{NbAs}$ develops an amorphous arsenicdepleted surface layer, as arsenic is preferentially removed during the FIB process, leaving behind a niobium (or in our case tantalum) rich layer that is superconducting ${ }^{26}$. This superconductivity is fully suppressed above $2 \mathrm{~T}$ and $1.5 \mathrm{~K}$, and thus does not affect the majority of the data presented.

Although the presence of a conducting surface layer changes the shapes of our resistivity curves at high fields, it does not alter any of the features from which we draw our main conclusions: the positions of the quantum limits for the various pockets, the onset of the resitivity increase in $\rho_{z z}$ near $50 \mathrm{~T}$, the temperature dependence of the increase in $\rho_{z z}$, the lack of a strong increase near $50 \mathrm{~T}$ in $\rho_{x x}$, and the sharp downturn in $\rho_{x x}$ near $80 \mathrm{~T}$ below $4 \mathrm{~K}$. The consistency of the ultrasound with the features in the transport provides further confirmation of the intrinsic nature of these features, as there was no FIB lithography performed on the ultrasound sample.

Fits to the conductivity. We fit the conductivity $\sigma_{z z}=\left(1 / \rho_{z z}\right)$ at each field slice as a function of temperature to a simple mode: a background $\sigma_{b}$ plus an activated component

$$
\sigma_{z z}=\sigma_{b}+\sigma_{0} e^{-\alpha / T}
$$

where $\alpha$ is the gap in units of kelvin. These fits plus the field-dependent fit parameters are shown in Supplementary Fig. 2.

In order to fit the gap down to the lowest possible field we normalized the conductivity to its value at $47 \mathrm{~T}$. Supplementary Fig. 2 demonstrates that while this procedure changes $\sigma_{b}$ and $\sigma_{0}$ it leaves $\Delta$ relatively unchanged. This is because the exponential factor dominates the fit, producing variability in $\sigma_{b}$ and $\sigma_{0}$ that depend on how (which field value, for example) the normalization is carried out but leaving $\Delta$ procedure-independent. While $\sigma_{0}$ is a physical parameter representing background conductivity in TaAs, the extracted values of $\sigma_{b}$ and $\sigma_{0}$ should not be taken as intrinsic.

Quantum oscillation analysis. According to the detailed quantum oscillation studies performed by Arnold et al. ${ }^{29}$, TaAs contains three distinct types of Fermi surface: electron-like W1 Weyl surfaces, electron-like W2 Weyl surfaces, and holelike trivial surfaces. We do not attempt to re-create their entire analysis here, but instead focus on the oscillations we observe in the $\mathbf{J}\|\mathbf{B}\| \mathbf{c}$ transport geometry. A complete treatment of the oscillation frequencies requires a full angle dependence which we have not measured: the purpose of the following analysis is to obtain a reasonable estimate for the quasiparticle lifetime $\tau$ for the Weyl electrons and to provide further evidence that the $\approx 18 \mathrm{~T}$ oscillation frequency is indeed coming from the same hole pocket identified by Arnold et al. ${ }^{29}$.

The full $\hat{\mathbf{c}}$-axis conductivity, including both background and oscillatory contributions, can be written at fixed angle as

$$
\sigma_{z z}=\sum_{i} \sigma_{0}^{i}(B)\left(1+\sum_{p} A^{i, p} R_{T}^{i, p} R_{D}^{i, p} \cos \left(2 \pi p \frac{F_{i}}{B}+\phi_{i}\right)\right),
$$

where the first sum is over the $i$ distinct Fermi surfaces, the sum $p$ is over the harmonics, $\sigma_{0}^{i}(B)$ are the field-dependent background conductivities, $A^{i, p}$ are amplitude factors, $F_{i}$ are the oscillation frequencies (proportional to the Fermi surface cross sectional area), and $\phi_{i}$ are phase factors ${ }^{51}$. The temperature factor is given by

$$
R_{T}^{i, p}=\frac{\frac{2 \pi^{2} p k_{B} T}{\hbar \omega_{c}^{i}}}{\sinh \left(\frac{2 \pi^{2} p k_{B} T}{\hbar \omega_{c}^{i}}\right)}
$$


where $\omega_{\mathrm{c}}^{i}=e B / m_{i}^{*}$ is the cyclotron frequency for effective mass $m_{i}^{*}$. The Dingle factor is

$$
R_{D}^{i, p}=e^{-\frac{\pi p}{\omega_{c}^{i} \tau_{i}}}
$$

where $\tau_{i}$ is the quasiparticle lifetime

It is important to note that the background conductivities in eq:osc are multiplicative with the oscillation amplitudes, not additive. Thus if a piece of Fermi surface does not contribute to $\sigma_{z z}$ then its oscillations will not be visible in $\rho_{z z}$, even if the quasiparticle lifetime for that surface is long. The W1 pockets are roughly elongated ellipses (see Fig. $3 \mathrm{c}$ of Arnold et al. ${ }^{29}$ ) with an aspect ratio of approximately 10:1 with the long axis oriented along the $\hat{\mathbf{c}}$ direction. With an inplane Fermi velocity of $3 \times 10^{5} \mathrm{~m} / \mathrm{s}$ these surfaces dominate the $\rho_{x x}$ transport and produce strong Shubnikov-de Haas oscillations with $\mathbf{B} \| \mathbf{c}$. For the $\rho_{z z}$ transport, however, we expect their Fermi velocity along $\hat{\mathbf{c}}$ to be reduced by the aspect ratio (a factor of 10 , so $v_{z} \approx 3 \times 10^{4} \mathrm{~m} / \mathrm{s}$ ), and thus contribute very little to the $\rho_{z z}$ transport. The W2 Weyl surfaces, on the other hand, are roughly isotropic in aspect ratio and have $v_{z} \approx 1.6 \times 10^{5} \mathrm{~m} / \mathrm{s}$; the hole pocket has $v_{z} \approx 7 \times 10^{5} \mathrm{~m} / \mathrm{s}$. As conductivity is proportional to Fermi velocity squared, $\rho_{z z}$ should be dominated by the W2 electron and trivial hole pockets. Note that the oscillations from a particular pocket can still be small due to other factors: for example, the hole pockets for $B \| \hat{\mathbf{c}}$ have a very unfavourable curvature factor that reduces their oscillation amplitude considerably (oscillation amplitudes scale as $1 / \sqrt{\partial^{2} A / \partial \kappa^{2}}$, where $A$ is the extremal area of the Fermi surface perpendicular to the magnetic field and $\kappa$ is the momentum direction parallel to the magnetic field) ${ }^{51}$.

We forgo the usual Fourier analysis of our data which can be difficult when there are only a few oscillations ${ }^{52}$. We instead perform a full three-dimensional fit to eq:osc for our data at temperatures where superconductivity is suppressed ( $T=$ $2.6,4,10$ and $20 \mathrm{~K}$ ). We can account for most features in the data fitting with $F_{w 2}=$ $6.5 \mathrm{~T}, F_{H}=17.2 \mathrm{~T}, m_{\mathrm{W} 2}^{*}=0.1 m_{\mathrm{e}}, m_{\mathrm{H}}^{*}=0.2 m_{\mathrm{e}}, \tau_{\mathrm{W} 2}=0.6 \mathrm{ps}$, and $\tau_{\mathrm{H}}=0.4 \mathrm{ps}$ Supplementary Fig. 5 . Values of $F_{\mathrm{W} 2}=5.5 \mathrm{~T}$ and $m_{\mathrm{W} 2}^{*}=0.105 m_{\mathrm{e}}$ were obtained by Arnold:2016 for the W2 Weyl pocket measuring magnetic torque with field along the â direction: given the relatively isotropic nature of this pocket we believe that we are observing the same piece of Fermi surface. Values of $F_{\mathrm{H}}=18.8 \mathrm{~T}$, and $\tau_{\mathrm{H}}=0.11 \mathrm{ps}$ were obtained for the hole pocket by Arnold et al. ${ }^{29}$, which again are in reasonable agreement with our own. Note that Arnold et al. ${ }^{29}$ were unable to obtain a mass for the hole pocket for $\mathbf{B} \| \mathbf{c}$ but predicted a mass of $m_{\mathrm{H}}^{*}=0.17 m_{\mathrm{e}}-$ very close to our experimentally determined $m_{\mathrm{H}}^{*}=0.2 m_{\mathrm{e}}$ for this pocket.

Pulse echo ultrasound. Pulse echo ultrasound measures the speed of sound $v$ transmitted through a sample for a specified sound propagation direction and polarization. Elastic moduli can then be computed via the relation $v=\sqrt{\frac{c}{\rho}}$,

although care must be taken when choosing the propagation and polarization directions if the relationship between $v$ and any particular $c_{i j}$ is to be kept simple (see Brugger et al. ${ }^{53}$ ). The measurement typically involves generating a short burst of ultrasound (of order a few hundred nanoseconds) via a piezoelectric transducer, and then recording the echoes with the same transducer as the sound travels back and forth between the faces of the sample parallel to the transducer face. The phase shift between each successive echo is related to the travel time (and hence sound velocity) of the sound pulse, and the amplitude decay with successive echoes is related to the ultrasonic attenuation.

Our implementation of pulse echo ultrasound was similar to Suslov et $\mathrm{al}^{54}$. with one notable exception: we forwent the mixing stage and directly recorded the full ultrasonic waveform with a $6 \mathrm{GS} / \mathrm{s}$ digitizer card (GaGe EON Express). This has two major advantages: it eliminates a large fraction of the electronics; and it allows the use of digital filtering and lock-in techniques that can be optimized and reprocessed after the experiment is completed.

We generated $315 \mathrm{MHz}$ longitudinal ultrasound with a $36^{\circ} \mathrm{Y}$-cut $\mathrm{LiNbO}_{3}$ transducer affixed to a $(0,0,1)$ face of a TaAs sample (Supplementary Fig. 6). As both the direction of propagation and the polarization are along $\hat{\mathbf{c}}$, we probe purely the $c_{33}$ elastic modulus in this tetragonal $\mathrm{crystal}^{53}$. The full field dependence at selected temperatures between 0.55 and $20 \mathrm{~K}$ is shown in Supplementary Fig. 6 . The velocity $v$ is calculated from the phase shift $\phi$ between successive echoes as

$$
\Delta v=-2 \pi f l \frac{\Delta \phi}{\phi^{2}}
$$

where $f$ is the ultrasonic frequency and $l$ is twice the sample length. The absolute phase $\phi$ can be obtained from the absolute spacing between successive echoes (a less accurate measure of the sound velocity, but the only way to get the absolute value) via $v=2 \pi f l / \phi$. All plotted velocity shifts are normalized to the phase shift at $B=0$ : the absolute shift as a function of temperature was not obtained.

Tight-binding model for TaAs. The primitive unit cell of TaAs consists of four sublattices, two Ta atoms and two As atoms, per primitive unit cell. The lattice structure has two mirror symmetries $M_{x}$ and $M_{y}$, TRS, and is invariant under $C_{4}$ rotation followed by a translation along the $\hat{\mathbf{z}}$ axis. We consider a threedimensional tight-binding model representing the TaAs lattice structure.
Specifically, we consider one spinful orbital per lattice site, giving rise to eight bands in total.

Our tight binding model:

$$
H=t \sum_{\langle i j\rangle, s} c_{i s}^{\dagger} c_{j s}+\sum_{i} \Delta_{i} c_{i}^{\dagger} c_{i}+i \lambda \sum_{\langle\langle i k\rangle\rangle, s, s^{\prime}} c_{i s}^{\dagger} c_{k s^{\prime}} \sum_{j}\left[\hat{\mathbf{d}}_{i j k} \cdot \sigma_{s s^{\prime}}\right]
$$

where $t$ is the nearest neighbor hopping; $\Delta$ is a staggered potential: $\Delta_{i}= \pm \Delta$ depending on whether the lattice site contains Ta or As; $\lambda$ is the amplitude of the spin orbit interaction between the next nearest neighbors; $s=\uparrow, \downarrow$ is the spin; and $\sigma$ 's are the corresponding Pauli matrices. $\hat{\mathbf{d}}_{i j k}$ is the unit vector in the direction of $\mathbf{d}_{i j} \times \mathbf{d}_{j k}$, where $j$ is an intermediate site between $i$ and $k$. This Hamiltonian preserves time reversal symmetry.

TaAs has 24 Weyl points, of which 8 are in the $k_{\mathrm{z}}=0$ plane (W1) and the rest 16 (W2) are at finite $k_{z}{ }^{7}$. The 8-fold (16-fold) multiplicity of the W1 (W2) Weyl points are guaranteed by model symmetries (lattice and TRS). More generally, the total number of Weyl points has to be $8_{n 1}+16_{n 2}$, where $n_{1}$ and $n_{2}$ are the numbers of sets of W1 and W2 Weyl points, respectively. For example, we will show the minimal tight-binding model can give 8 Weyl points, while adding extra terms and anisotropy reproduces the 24 Weyl points.

A finite $\Delta$ in Eq. 7 explicitly breaks inversion symmetry, thus is vital to the emergence of Weyl semimetals. On the other hand, in the limit of $\Delta \rightarrow \infty$ the system has to be a trivial insulator. In the presence of TRS, a Weyl semimetal is guaranteed to exist between a topological insulator and a trivial insulator ${ }^{55}$. During the evolution, pairs of Weyl points are generated, transported across the momentum space, and re-annihilate in different pairs, giving rise to a change of the $\mathbf{Z}_{2}$ topological index. We note that the W2 Weyl points always change the topological index trivially by 2 , and it is up to the W1 Weyl points to change the $\mathbf{Z}_{2}$ topological index by a nontrivial 1 . This suggests that while the W1 Weyl points are essential and guaranteed to occur somewhere during the interpolation, the W2 Weyl points are less fundamental and involve more fine-tuning.

First, we show that the Hamiltonian in Eq. 7 can give a Weyl semimetal in certain parameter regions. For model parameters $t=1.0, \Delta=0.7$, and $\lambda=0.1$, the dispersion around $\mathbf{k}=(0.7,2.56,0)$ is shown in Supplementary Fig. 7 . The band touching at a single Weyl point and the nearby linear dispersion are clearly established. The locations of all the eight Weyl points within the momentum space are illustrated in Supplementary Fig. 8. Being at $k_{z}=0$, these Weyl points belong to the W1 group. Interestingly, we observe the shift and annihilation of the Weyl nodes as we change the amplitude $\Delta$ of inversion symmetry breaking. We also note that the dispersion between the pair of adjacent Weyl nodes resembles that observed in band-structure calculations (Supplementary Fig. 9) ${ }^{25}$.

A comparison can be made between the electronic structure of the minimum tight-binding model Supplementary Fig. 10 and that of the actual TaAs crystal from band-structure calculations (see for example Fig. $4 \mathrm{c}$ from ref. ${ }^{23}$ ). The minimum model in Eq. 7 is already capable of qualitatively resembling the ab-initio band structures near the chemical potential.

We now consider the fate of the Weyl physics in the presence of an external magnetic field. For simplicity, we first focus on the situation when the magnetic field is along the $\mathrm{c}$ axis: $\mathbf{B}=B_{z} \hat{\mathbf{z}}$. Since $k_{\mathrm{z}}$ remains a good quantum number, together with the quantization within the $k_{x}-k_{\mathrm{y}}$ plane, gives rise to Landau bands. For an individual Weyl node, there exists a chiral Landau band, whose up-moving or down-moving is associated with the chirality of the original Weyl node. However, as discussed in other theoretical studies, this physics becomes challenged at large magnetic fields ${ }^{37,38}$.

Semi-classically, magnetic fields introduce finite resolution in $k_{x}-k_{y}$ momentum space, inversely proportional to the magnetic length. Therefore, at large enough $B$, the pair of separated Weyl nodes with opposite chirality becomes indistinguishable and magnetic breakdown makes tunneling between them nonnegligible. This was addressed schematically in refs. ${ }^{37,38}$. Here, we will study from the perspective of our tight-binding model in Eq. 7. We find an emergent gap whose amplitude and onset field are roughly consistent with those observed in experiments for Weyl node separation $\delta k \sim 0.15 \pi / a$, which is at least a factor of 2 larger than DFT results ${ }^{23,25}$ yet can be attributed to a relatively small modification to the overall band structure. Since the separation between the pair of adjacent Weyl fermions are small, they are very sensitive to small shifts in bandstructure which may be beyond the resolution capability of the DFT calculations. We also note that the Weyl nodes separation $\delta k \sim 0.15 \pi / a$ is consistent with the position of the Weyl nodes in the surface Brillouin zone observed in ARPES experiments ${ }^{27}$. As most studies have found W2 Weyl fermions to have larger separations than the W1 Weyl fermions, the former should be more resilient and dominates the fate of the Weyl physics in large magnetic fields. Although the W2 Weyl nodes are away from the $k_{z}=0$ plane, it only causes a constant shift to the zero of the $k_{z}$ good quantum number and does not change the following discussion. The $k$-space distance between different pairs of Weyl nodes is much greater than the intra-pair separation and quantum tunneling due to magnetic breakdown between different pairs can be neglected.

To begin with, we neglect the Zeeman effect, and the vector potential of the magnetic field is chosen as $\mathbf{A}=(0, B x, 0)$. We also set Fermi velocities in the $v_{\mathrm{F}}^{x} \sim v_{\mathrm{F}}^{y} \sim 291000 \mathrm{~m} / \mathrm{s}=1.92 \mathrm{eV} \cdot \AA$ observed via quantum oscillation measurements. These, together with the Weyl node separation $\delta k \sim 0.15 \pi / a$ and $a=3.437 \AA$ in TaAs allows us to set proper units for our numerical results. 
A typical Landau band dispersion is illustrated in Supplementary Fig. 11. Each band is four-fold degenerate, suggesting the tunneling between different pairs of Weyl nodes are still negligible even at a large magnetic field $B_{z}=145 \mathrm{~T}$. On the other hand, a gap is clearly observable between the $n=0$ chiral Landau bands.

An important question is how does the size of this anti-crossing gap depends on the applied magnetic field. Intuitively, the gap should be exponentially suppressed at smaller field $l_{B} \delta_{k} \gg 1$, and scale linearly at larger magnetic field. Such behavior is clearly observed in Supplementary Fig. 12 and Supplementary Fig. 13, where the units of the data are interpreted for TaAs W2 Weyl nodes with separation $\delta k \sim 0.15 \pi / a$ and $\delta k \sim 0.165 \pi / a$, respectively.

Now we consider another potentially important ingredient-the Zeeman effect -particularly important because semi-metals and semi-conductors often give rise to large $g$-factors. For example, the $g$-factor of TaP has been estimated to be between 2 and 2.9 for one set of orbits and 5.5 and 6.7 for a second set ${ }^{56}$. To incorporate this we add an additional contribution to the Hamiltonian:

$$
H_{\text {Zeeman }}=-\mu_{\mathrm{B}} g S^{z}
$$

The results with and without the Zeeman effect are compared in Supplementary Fig. 14. The results suggest that though the impact of the Zeeman effect is relatively minor (Supplementary Fig. 14 upper panel), the quantitative change to the anticrossing feature is visible especially for larger Weyl nodes separation $\delta k$ that allows weaker gaps with a larger magnetic field threshold, see Supplementary Fig. 14 lower panel.

The influence of the Zeeman effect can be understood via its modifications to the band structure in the absence of the vector potentials, see Supplementary Fig. 15. In the presence of Zeeman energy, the Weyl nodes moves further apart, making quantum tunneling between the pair of Weyl nodes more difficult thus suppressing the anti-crossing gap size.

Data Availability. The data that support the findings of this study are available from the corresponding author upon reasonable request.

Received: 4 March 2018 Accepted: 3 May 2018

Published online: 07 June 2018

\section{References}

1. Kamerlingh Onnes, H. The superconductivity of mercury. Comm. Phys. 122, 124 (1911).

2. George Bednorz, J. \& Alex Müller, K. Possible high-Tc superconductivity in the Ba-La-Cu-O system. Z. für Phys. B Condens. Matter 64, 189-193 (1986).

3. Kivelson, S. A. et al. Electronic liquid-crystal phases of a doped Mott insulator. Nature 393, 550-553 (1998).

4. Tsui, D. C., Stormer, H. L. \& Gossard, A. C. Two-dimensional magnetotransport in the extreme quantum limit. Phys. Rev. Lett. 48, 1559-1562 (1982).

5. Mott, N. F. Metal-insulator transition. Rev. Mod. Phys. 40, 677 (1968).

6. Wan, X., Turner, A. M., Vishwanath, A. \& Savrasov, SergeyY. Topological semimetal and Fermi-arc surface states in the electronic structure of pyrochlore iridates. Phys. Rev. B 83, 205101 (2011)

7. Weng, H., Fang, C., Fang, Z., Bernevig, B. A. \& Dai, Xi Weyl semimetal phase in noncentrosymmetric transition-metal monophosphides. Phys. Rev. X $\mathbf{5}$, 011029 (2015).

8. Su-Yang Xu, Ilya et al. Discovery of a Weyl fermion semimetal and topological Fermi arcs. Science 349, 613-617 (2015).

9. Lv, B. Q. et al. Experimental discovery of Weyl semimetal TaAs. Phys. Rev. X 5, 031013 (2015).

10. Nielsen, H. B. \& Ninomiya., M. The Adler-Bell-Jackiw anomaly and Weyl fermions in a crystal. Phys. Lett. B 130, 389-396 (1983).

11. Wei, H., Chao, S. P. \& Aji, V. Excitonic phases from Weyl semimetals. Phys. Rev. Lett. 109, 196403 (2012).

12. Xiao-Tian, Z. \& Ryuichi, S. Transport properties of density wave phases in three-dimensional metals and semimetals under high magnetic field. Phys. Rev. B 95, 205108 (2017).

13. Cho, G. Y., Bardarson, J. H., Lu, Y. M. \& Moore., J. E. Superconductivity of doped Weyl semimetals: Finite-momentum pairing and electronic analog of the ${ }^{3} \mathrm{He}-A$ phase. Phys. Rev. B 86, 214514 (2012).

14. Meng, T. \& Balents, L. Weyl superconductors. Phys. Rev. B 86, 054504 (2012).

15. Zhang, X. X. \& Nagaosa., N. Tomonaga-Luttinger liquid and localization in Weyl semimetals. Phys. Rev. B 95, 205143 (2017).

16. Armitage, N. P., Mele, E. J. \& Vishwanath, A. Weyl and Dirac semimetals in three-dimensional solids. Rev. Mod. Phys. 90, 015001 (2018).

17. Adler, S. L. Axial-vector vertex in spinor electrodynamics. Phys. Rev. 177, 2426-2438 (1969).
18. Bell, J. S. \& Jackiw, R. A PCAC puzzle: $\pi 0 \gamma \gamma$ in the $\sigma$-model. Il Nuovo Cim. A (1965-1970) 60, 47-61 (1969).

19. Son, D. T. \& Spivak, B. Z. Chiral anomaly and classical negative magnetoresistance of Weyl metals. Phys. Rev. B 88, 104412 (2013)

20. Spivak, B. Z. \& Andreev, A. V. Magnetotransport phenomena related to the chiral anomaly in Weyl semimetals. Phys. Rev. B 93, 085107 (2016).

21. A. Lucas, R. A Davison, and S. Sachdev. Hydrodynamic theory of thermoelectric transport and negative magnetoresistance in Weyl semimetals. Proc. Natl Acad. Sci. 113, 9463-9468 (2016).

22. Pavan, H. \& Xiao-Liang, Qi Tunable circular dichroism due to the chiral anomaly in Weyl semimetals. Phys. Rev. B 91, 081106 (2015).

23. Huang, $\mathrm{Xi}$ et al. Observation of the chiral-anomaly-induced negative magnetoresistance in 3D Weyl semimetal TaAs. Phys. Rev. X 5, 031023 (2015).

24. Johannes Gooth, A. C. et al. Experimental signatures of the mixed axial-gravitational anomaly in the Weyl semimetal NbP. Nature 547, 324 (2017).

25. Ma, Q. et al. Direct optical detection of Weyl fermion chirality in a topological semimetal. Nat. Phys. 13, 842 (2017).

26. Bachmann, M. D. et al. Inducing superconductivity in Weyl semimetal microstructures by selective ion sputtering. Sci. Adv. 3, e1602983 (2017).

27. Lv, B. Q. et al. Observation of Weyl nodes in TaAs. Nat. Phys. 11, 724-727 (2015).

28. Zhang, C. L. et al. Electron scattering in tantalum monoarsenide. Phys. Rev. B 95, 085202 (2017)

29. Arnold, F. et al. Chiral Weyl pockets and fermi surface topology of the weyl semimetal TaAs. Phys. Rev. Lett. 117, 146401 (2016).

30. C. L. Zhang, et al. Signatures of the Adler-Bell-Jackiw chiral anomaly in a Weyl fermion semimetal. Nat. Commun. 7, 10735 (2016).

31. P. J. W. Moll, et al. Magnetic torque anomaly in the quantum limit of Weyl semimetals. Nat. Commun. 7, 12492 (2016)

32. Arnold, F. et al. Negative magnetoresistance without well-defined chirality in the Weyl semimetal TaP. Nat. Commun. 7, 11615 (2016).

33. Pippard, Alfred B. Magnetoresistance in Metals, Vol. 2. (Cambridge University Press, Cambridge, U K, 1989)

34. R. D. dos Reis, et al. On the search for the chiral anomaly in Weyl semimetals: the negative longitudinal magnetoresistance. New J. Phys., 18, 2016. ISSN 1367-2630. https://doi.org/10.1088/1367-2630/18/8/085006

35. Argyres, P. N. \& Adams, E. N. Longitudinal magnetoresistance in the quantum limit. Phys. Rev. 104, 900 (1956).

36. Sergei Stanislavovich Murzin. Electron transport in the extreme quantum limit in applied magnetic field. Phys.-Uspekhi 43, 349-364 (2000).

37. Kim, P., Ryoo, Ji. H. \& Park, C. H. Breakdown of the chiral anomaly in Weyl semimetals in a strong magnetic field. Phys. Rev. Lett. 119, 266401 (2017).

38. Chan, C. K. \& Lee, P. A. Emergence of gapped bulk and metallic side walls in the zeroth Landau level in Dirac and Weyl semimetals. Phys. Rev. B 96, 195143 (2017).

39. Zhang, C. L. et al. Magnetic-tunnelling-induced Weyl node annihilation in TaP. Nat. Phys. 13, 979-986 (2017).

40. Popovi, D., Fowler, A. B. \& Washburn., S. Metal-insulator transition in two dimensions: effects of disorder and magnetic field. Phys. Rev. Lett. 79, 1543 (1997).

41. Kravchenko, S. V., Kravchenko, G. V., Furneaux, J. E., Pudalov, V. M. \& dIorio., M. Possible metal-insulator transition at $\mathrm{B}=0$ in two dimensions. Phys. Rev. B 50, 8039 (1994).

42. S. Tanuma, et al. Physics in high magnetic fields. Springer Series in Solid State Sciences (1981).

43. Fauqué, B. et al. Two Phase Transitions Induced by a Magnetic Field in Graphite. Phys. Rev. Lett. 110, 266601 (2013).

44. Zengwei Zhu, R. D. et al. Magnetic field tuning of an excitonic insulator between the weak and strong coupling regimes in quantum limit graphite. Sci. Rep. 7, 1733 (2017).

45. Pippard, A. B. Ultrasonic attenuation in metals. Philos. Mag. 46, 1104-1114 (1955). ISSN 0031-8086.

46. LeBoeuf, D. et al. Thermodynamic signatures of the field-induced states of graphite. Nat. Commun. 8, 1337 (2017).

47. Spivak, B. \& Kivelson., S. A. Transport in two dimensional electronic microemulsions. Ann. Phys. 321, 2071-2115 (2006).

48. Paalanen, M. A. et al. RF conductivity of a two-dimensional electron system at small Landau-level filling factors. Phys. Rev. B 45, 11342-11345 (1992).

49. Shekhter, A. et al. Bounding the pseudogap with a line of phase transitions in

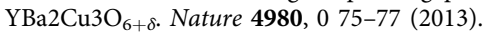

50. Field, S. B. et al. Evidence for depinning of a Wigner crystal in $\mathrm{Hg}$-Cd-Te. Phys. Rev. B 33, 5082-5085 (1986).

51. D. Shoenberg. Magnetic Oscillations in Metals. (Cambridge University Press, Cambridge, U K, 1984). ISBN 9780521224802.

52. Ramshaw, B. J. et al. Angle dependence of quantum oscillations in $\mathrm{YBa}_{2} \mathrm{Cu}_{3} \mathrm{O}_{6.59}$ shows free-spin behaviour of quasiparticles. Nat. Phys. 7 , 234-238 (2011). 
53. Brugger, K. Pure modes for elastic waves in crystals. J. Appl. Phys. 36, 759-768 (1965).

54. Suslov, A. et al. Ultrasonic instrumentation for measurements in high magnetic fields. II. Pulsed magnetic fields. Rev. Sci. Instrum. 77, 035105 (2006).

55. Turner, A. M., Zhang, Yi, Mong, R. S. K. \& Vishwanath., A. Quantized response and topology of magnetic insulators with inversion symmetry. Phys. Rev. B 85, 165120 (2012).

56. $\mathrm{Hu}, \mathrm{J}$. et al. $\pi$ Berry phase and Zeeman splitting of Weyl semimetal TaP. Sci. Rep. 6, 18674 (2016).

\section{Acknowledgements}

This work was performed at the National High Magnetic Field Laboratory, which is supported by National Science Foundation Cooperative Agreement No. DMR-1157490 and the State of Florida. E.D.B, F.R., and R.D.M. acknowledge funding from the LANL LDRD DR20160085 'Topology and Strong Correlations'. B.J.R. acknowledges funding from LANL LDRD 20160616ECR 'New States of Matter in Weyl Semimetals'. M.K.C. acknowledges funding from the U.S. Department of Energy Office of Basic Energy Sciences Science at $100 \mathrm{~T}$ program. P.J.M. is supported by the Max-Planck-Society and the European Research Council (ERC) under the European Union's Horizon 2020 research and innovation programme (grant agreement No. 715730). M.D.B. acknowledges studentship funding from EPSRC under Grant No. EP/I007002/1. Y.Z. was supported in part by NSF DMR-1308089 and E.A.K. was supported in part by National Science Foundation (Platform for the Accelerated Realization, Analysis, and Discovery of Interface Materials (PARADIM)) under Cooperative Agreement No. DMR-1539918.

\section{Author contributions}

B.J.R. and R.D.M. conceived of the experiment, E.D.B., N.J.G., and F.R. grew the samples, B.J.R. and R.D.M. performed the focused ion beam lithography, B.J.R., J.B.B., A.M., F.B., and R.D.M. designed and built the experiment, B.J.R., K.A.M., P.J.W.M., M.B., M.K.C., J.B.B., and R.D.M. performed the experiment, B.J.R. and K.A.M. analyzed the data, Y.Z. and E.A.K. developed the tight-binding model and provided theoretical guidance, and B.J.R. wrote the manuscript with input from all coauthors.

\section{Additional information}

Supplementary Information accompanies this paper at https://doi.org/10.1038/s41467018-04542-9.

Competing interests: The authors declare no competing interests.

Reprints and permission information is available online at http://npg.nature.com/ reprintsandpermissions/

Publisher's note: Springer Nature remains neutral with regard to jurisdictional claims in published maps and institutional affiliations.

(c) Open Access This article is licensed under a Creative Commons Attribution 4.0 International License, which permits use, sharing, adaptation, distribution and reproduction in any medium or format, as long as you give appropriate credit to the original author(s) and the source, provide a link to the Creative Commons license, and indicate if changes were made. The images or other third party material in this article are included in the article's Creative Commons license, unless indicated otherwise in a credit line to the material. If material is not included in the article's Creative Commons license and your intended use is not permitted by statutory regulation or exceeds the permitted use, you will need to obtain permission directly from the copyright holder. To view a copy of this license, visit http://creativecommons.org/ licenses/by/4.0/.

(C) The Author(s) 2018 